\title{
Geometrical Aberration Suppression for Large Aperture Sub-THz Lenses
}

\author{
M. $\operatorname{Rachon}^{1}$ (D) K. Liebert ${ }^{1} \cdot$ A. Siemion ${ }^{1} \cdot$ J. Bomba $^{1} \cdot$ \\ A. Sobczyk $^{1}$ - W. Knap ${ }^{2}$ - D. Coquillat ${ }^{2}$. J. Suszek ${ }^{1}$. \\ M. Sypek ${ }^{1}$
}

Received: 22 July 2016 / Accepted: 15 November 2016 / Published online: 30 November 2016

(C) The Author(s) 2016. This article is published with open access at Springerlink.com

\begin{abstract}
Advanced $\mathrm{THz}$ setups require high performance optical elements with large numerical apertures and small focal lengths. This is due to the high absorption of humid air and relatively low efficiency of commercially available detectors. Here, we propose a new type of double-sided sub-THz diffractive optical element with suppressed geometrical aberration for narrowband applications $(0.3 \mathrm{THz})$. One side of the element is designed as thin structure in non-paraxial approach which is the exact method, but only for ideally flat elements. The second side will compensate phase distribution differences between ideal thin structure and real volume one. The computer-aided optimization algorithm is performed to design an additional phase distribution of correcting layer assuming volume designing of the first side of the element. The experimental evaluation of the proposed diffractive component created by $3 \mathrm{D}$ printing technique shows almost two times larger performance in comparison with uncorrected basic diffractive lens.
\end{abstract}

Keywords Sub-terahertz $\cdot$ Double-sided lens $\cdot 3$ D printing $\cdot$ Diffractive lens $\cdot$ Kinoform $\cdot$ Narrowband

\section{Introduction}

$\mathrm{THz}$ beam shaping has vast applications in modern technology. Depending on the application and the material used for manufacturing, it can be practically realised as refractive elements [1], reflective mirror surfaces [2], diffractive structures [3] or

M. Rachon

rachon@if.pw.edu.pl

1 Faculty of Physics, Warsaw University of Technology, Warsaw, Poland

2 Charles Coulomb Laboratory (L2C) - UMR5221 CNRS - University Montpellier, Montpellier, France 
structures including metamaterials [4]. Focusing on diffractive elements that are thinner require smaller amount of material, are easier in positioning and are lighter, we can generally consider two main types of applications of designed diffractive optical elements (DOEs). The first one describes the beam shaping providing the expected amplitude and phase distributions at a particular plane in space [5]. It can be often used in $\mathrm{THz}$ systems with active illumination. Another type of elements can be generally considered as energy concentrators on different kinds of detectors. Moreover, for detector matrices, a special imaging process is often used. Energy concentration or imaging can be realised for both active and passive illumination.

The next aspect, that should be analysed, concerns spectral characteristics of manufactured element for the performed experiment. Many devices working in active mode have narrowband characteristics. Typically, the exact frequency of the $\mathrm{THz}$ beam is assumed. The narrowband operation is mostly realised by detectors (narrow spectral line or heterodyne detection). Moreover, using standard diffractive optical elements introducing phase retardation of $2 \pi$ limits their application to narrowband operation. However, advanced diffractive optics (applied also in $\mathrm{THz}$ range) has several advantages, for example, suppressing the geometrical and chromatic aberration [6]. Moreover, such elements are relatively compact and thin, so the attenuation, the weight and the material cost is not significant [7]. In case of using a little bit thicker elements, when the phase retardation is a multiple of $2 \pi$ for the design wavelength (DWL), a high order kinoform structures are generated that may work in a wide frequency range [8-10], so suppressing also chromatic aberration in DOEs is possible.

Here, we limit our considerations to diffractive optics designed for narrowband applications. The analysed lens-like elements have their focal lengths equal to their diameters, which requires sophisticated non-paraxial designing process. We prove that not taking into account the real thickness of the designed DOE (which is the order of single $\mathrm{mm}$ in sub-THz range) introduces a significant phase error, which decreases the performance of the element. We show that the proper phase correction added on the opposite side of the element can significantly improve the efficiency of the diffractive optics. No matter what attenuation of the material we will assume, the errors occurring in the difference of designing thin and thick elements being only phase structures are causing decrease of the efficiency of the designed element.

\section{Theoretical Background}

We assume that the lens-like element is illuminated with a quasi-monochromatic plane wave; thus, we expect the diffraction limited focal spot [11] at a distance of the focal length behind the element. According to the theory, geometrical aberrations should enlarge the focal spot size and decrease its intensity. Moreover, in the case of the refractive element, its transmittance of $\mathrm{THz}$ radiation in the centre is significantly lower than in outer parts of the element. It results from larger thickness in the middle of the structure and therefore higher attenuation introduced by the medium, which leads to additional boost of geometrical aberrations [8, 11]. The simplest alternative to the refractive element for narrowband illumination is a diffractive one which is thin (no attenuation of medium) and in case of continuous phase profile (kinoform structure) reaches $100 \%$ of diffractive efficiency. 
In theoretical considerations, the lens can be represented by a complex transmittance under paraxial approximation [11] (small angles approximation, thus on-axis propagation) which in spherical coordinates can be described as:

$$
T(r)=e^{-\frac{i k r^{2}}{2 f}}
$$

where $k=2 \pi / \lambda$, is the wave number, $f$-focal length and the structure is assumed to be flat with maximal phase retardation of $2 \pi$.

In the case sub-THz optics, mostly the diameter of the element is almost equal to its focal length $f$ and then the complex transmittance is described with the equation in the non-paraxial approach [11] (large angles and off-axis propagation):

$$
T(r)=e^{-i k \sqrt{r^{2}+f^{2}}}
$$

where $d=f=50 \mathrm{~mm}$.

Numerical calculations lead to results presented in Fig. 1. The phase distribution of two diffractive elements designed as kinoform structures - one in paraxial approach (on-axis) and other in non-paraxial approach (off-axis) - is shown in Fig. 1a, b, respectively, while the difference between these two approaches is illustrated in Fig. 1c. Additionally, the diagrams of the phase value versus the distance from the middle are shown to illustrate the difference between two thin designing approaches. Notice that the subtraction of these two phase distributions is illustrated in Fig. 1c and it can be clearly seen that in the middle there is almost no difference between on- and off- axis approaches while on the edge this difference becomes significant. In Fig. 1, we can see the phase distributions (on the left) and their crosssections (on the right).

To suppress the geometrical aberration, we propose to add the correcting structure on one side of the manufactured element. It compensates the negative influence of the difference between the theoretical assumption that structure is totally flat and real manufacturing process that forms an element having particular thickness. The computer-aided optimization is performed to design a phase correction layer on the opposite side of the element designed in the non-paraxial regime.

(a) paraxial design
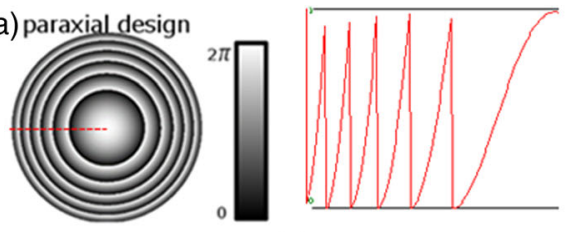

(b) non paraxial design
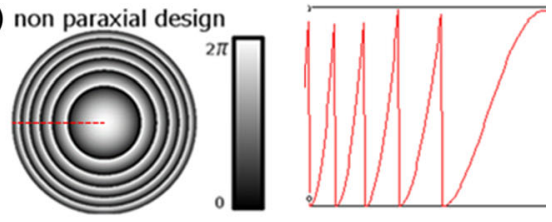

(c) phase difference
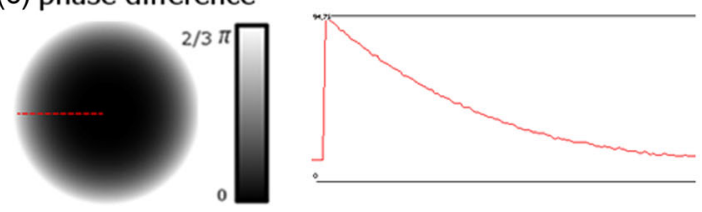

Fig. 1 The phase distribution for paraxial (a) and non-paraxial designs (b) together with their cross-sections. The difference between $\mathbf{a}$ and $\mathbf{b}$ is shown in $\mathbf{c}$ which corresponds to the phase difference. The lens diameter $d=50 \mathrm{~mm}$, focal length $f=50 \mathrm{~mm}$, design wavelength $\mathrm{DWL}=1 \mathrm{~mm}$. The black colour corresponds to $0 \pi$ phase shift, while white denotes $2 \pi$ phase shift 


\section{Numerical Modelling}

The calculation matrix used for numerical modelling was composed of $4096 \times 4096$ pixels with sampling period of $117 \mu \mathrm{m}$ in both directions. The design wavelength was assumed to be $1 \mathrm{~mm}$ (which corresponds to $0.3 \mathrm{THz}$ ). Each pixel corresponds to the complex number representing the amplitude and the phase distributions of the sample. The propagation algorithm was based on the wave optics approach which allows to maintain thick structure design. Taking into account both the refractive index and the attenuation of the material, the calculations were performed by dividing the real thickness of the structure $(1.56 \mathrm{~mm})$ into 32 parallel slices (each having the thickness of $48.75 \mu \mathrm{m}$ ). In the volume of the element, the light propagation was calculated using the modified beam propagation method (BPM) [12]. The transformation of the complex light field between slices was calculated using the non-paraxial approach of the modified convolution method (MCM) [13, 14].

The diffractive elements were manufactured by means of 3D printing from polyamide 12 (PA12) [5], which at the frequency of $0.3 \mathrm{THz}$ has the refractive index $n=1.64$ and the absorption coefficient $0.27 \mathrm{~cm}^{-1}$. The sampling of numerical modelling was adjusted to the voxel size of the machine used for 3D printing. The cost of the element depends on the time of working of the machine (depending on the volume of the structure), so structures having only one side are a little bit less expensive. Nevertheless, the cost of manufacturing single lens is dependent on its size and accuracy but in comparison with other techniques is cost-efficient (mostly the manufacturing of one lens is not exceeding 100 euro due to the low volume of the elements, typically it is much less due to the fact that price significantly decreases when multiple lenses are manufactured in single manufacturing process).

Here, three structures were analysed: kinoform lens designed in paraxial approximation, kinoform lens designed with non-paraxial approach and a structure that is the combination of non-paraxially designed kinoform lens on one side and advanced phase correcting element on the other. The correcting structure was calculated with new algorithm based on the comparison of the ideal thin structure phase distribution with the phase retardation introduced by real thick element (calculated by means of modified BPM). The algorithm enabling simulations of volume structures allows to predict the differences encountered during experimental evaluation of manufactured element in comparison with simulated thin theoretical element. The novelty can be described as the necessity of the phase correction added to the thin phase element (calculated as the difference with volume structure) to correct the phase distribution after the optical element to be ideal. It means that we do not only simulate volume structures, we do not only simulate thin structures, but we do the combined simulations (taking into account that the resulting element is actually a volume structure) to obtain ideal phase profile after the structure.

The algorithm illustrated in Fig. 2 determines the phase distribution of the correcting layer. In the first step, the phase distribution of the thin element $\varphi_{\text {ideal }}$ is calculated. Then, it is compared with the phase distribution of the element $\varphi_{\text {volume }}$ calculated as the volume structure taking into account the modified BPM. These two phase distributions are subtracted and complex coupled to obtain inverse phase distribution compensating the error. Considering phase transmittances of these structures we can write:

$$
\left[\left(e^{i \varphi_{\text {ideal }}}\right)^{*} \cdot e^{i \varphi_{\text {volume }}}\right]^{*}=\left[e^{i\left(\varphi_{\text {volume }}-\varphi_{\text {ideal }}\right)}\right]^{*}=e^{i \varphi_{\text {correction }}},
$$


(a)

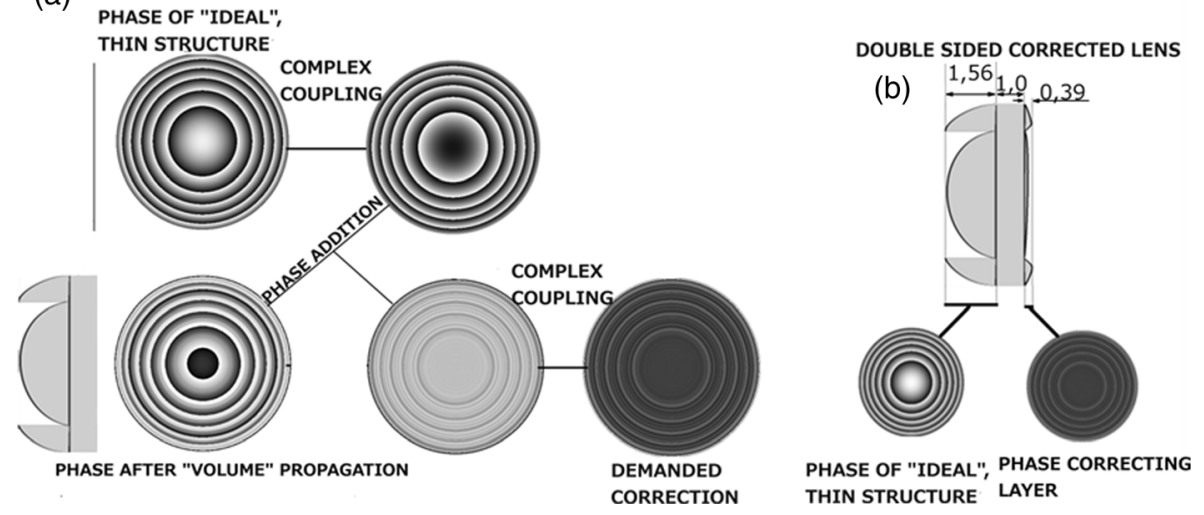

Fig. 2 An illustration of the corrected lens designing process (a) and the cross-section of the corrected doublesided lens with marked size (b). All circular images corresponds to phase distributions, where black colour means $0 \pi$ phase shift, while white denotes $2 \pi$ phase shift. Two cross-sections illustrate thicknesses of structures in $\mathrm{mm}$

where

$$
\begin{aligned}
& e^{i \varphi_{\text {ideal }}} \text { - phase transmittance of an ideal thin structure } \\
& e^{i \varphi_{\text {volume }}} \text {-phase transmittance after a volume propagation }
\end{aligned}
$$

and when we assume that all amplitudes are uniform and equal to unity.

The main assumption is to correct the difference between the phase distribution of the ideal converging kinoform lens and the phase distribution of the wavefront after propagation through the real thick structure. A thin phase structure is obtained from the Eq. (2). The initial phase distribution of volume structure was obtained after the propagation using modified beam propagation method through the volume medium. Then, in order to achieve the difference between the phase distribution of the thin and the thick structures, the numerical algorithm was designed (Fig. 2a). At first, the phase distribution of the ideal thin structure was complex conjugated and added to the phase distribution received after the propagation in the volume structure. In the next step, the complex conjugating of the phase transmittance was performed once again and this resulted in the phase distribution of correcting structure. Such structure was $3 \mathrm{D}$ printed on the back of the non-paraxially designed kinoform lens as shown in Fig. $2 \mathrm{~b}$. Applying such additional structure on the examined DOE significantly increased the efficiency of the kinoform structure.

\section{Experimental Results}

The experimental investigations were conducted in the optical system presented in Fig. 3. It consisted of tuneable VDI multiplier as a source, chopper connected to the lock-in system and mirror and parabolic mirror (PM) to create parallel beam. Then, this light was focused on a pinhole by additional lens and behind it formed a quasi-spherical THz wave. After propagation at $5 \mathrm{~m}$, we assumed to have almost parallel beam with relatively uniform power distribution that illuminated the tested structure. All data were captured by raster scanning with a single detector consisting of a Si MOSFET and a bow-tie antenna with a radius of $60 \mu \mathrm{m}$. The XY 


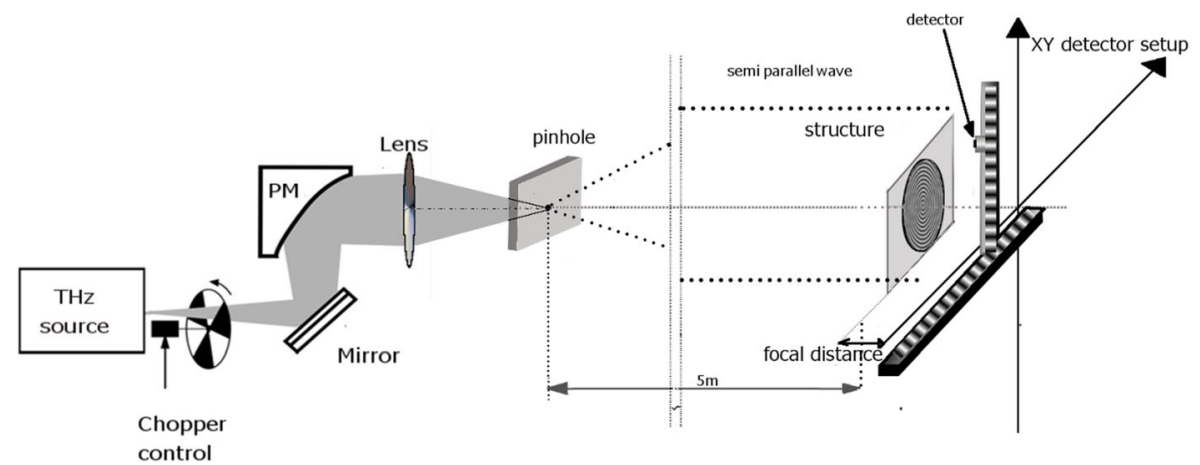

Fig. 3 The scheme of the experimental setup using fast and sensitive Si MOSFET detectors

plane was scanned at a focal distance behind the examined structure [15] with the resolution of $300 \mu \mathrm{m}$.

The experiment was carried out for three structures illustrated in Fig. 4-one-sided on-axis kinoform, one-sided off-axis kinoform and double-sided structure consisting of off-axis kinoform and correcting element. All scans were carried out in the same conditions at room temperature.

The experimental results are shown in Table 1 and are normalised to the value obtained for the paraxially designed kinoform lens (Fig. 4a). All intensity distributions have been presented as $2 \mathrm{D}$ diagrams and $3 \mathrm{D}$ plots in such a manner to enable comparison of the increase of the efficiency in both non-paraxially designed structures (3D plots) and to show that the focal spot remains small and close to the diffraction limit (2D intensity distributions). It is worth noticing that the noise is significantly smaller for the off-axis kinoform structure with correcting layer (Table 2). The noise level was calculated as the standard deviation of pixels' values in the background area.

(a)

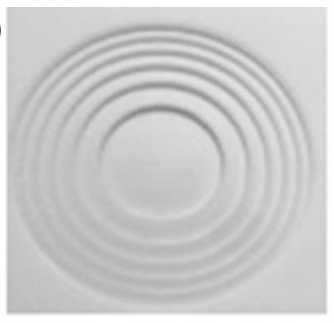

(b)

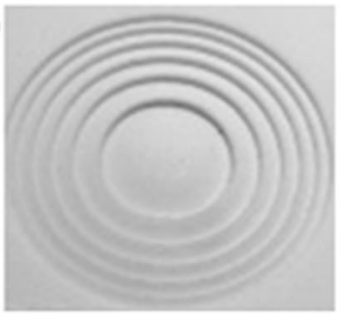

(c)
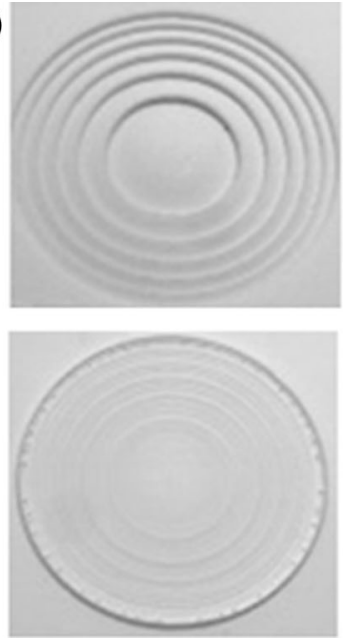

Fig. 4 The photographs of the paraxial kinoform lens (a), the non-paraxial kinoform lens (b) and the doublesided structure (c) consisting of the non-paraxial kinoform lens (top) and the correcting structure (bottom) 
Table 1 The experimental results for three structures: the kinoform designed in the paraxial approximation, the kinoform designed in the non-paraxial approximation and the double-sided kinoform with non-paraxial lens with additional correcting structure

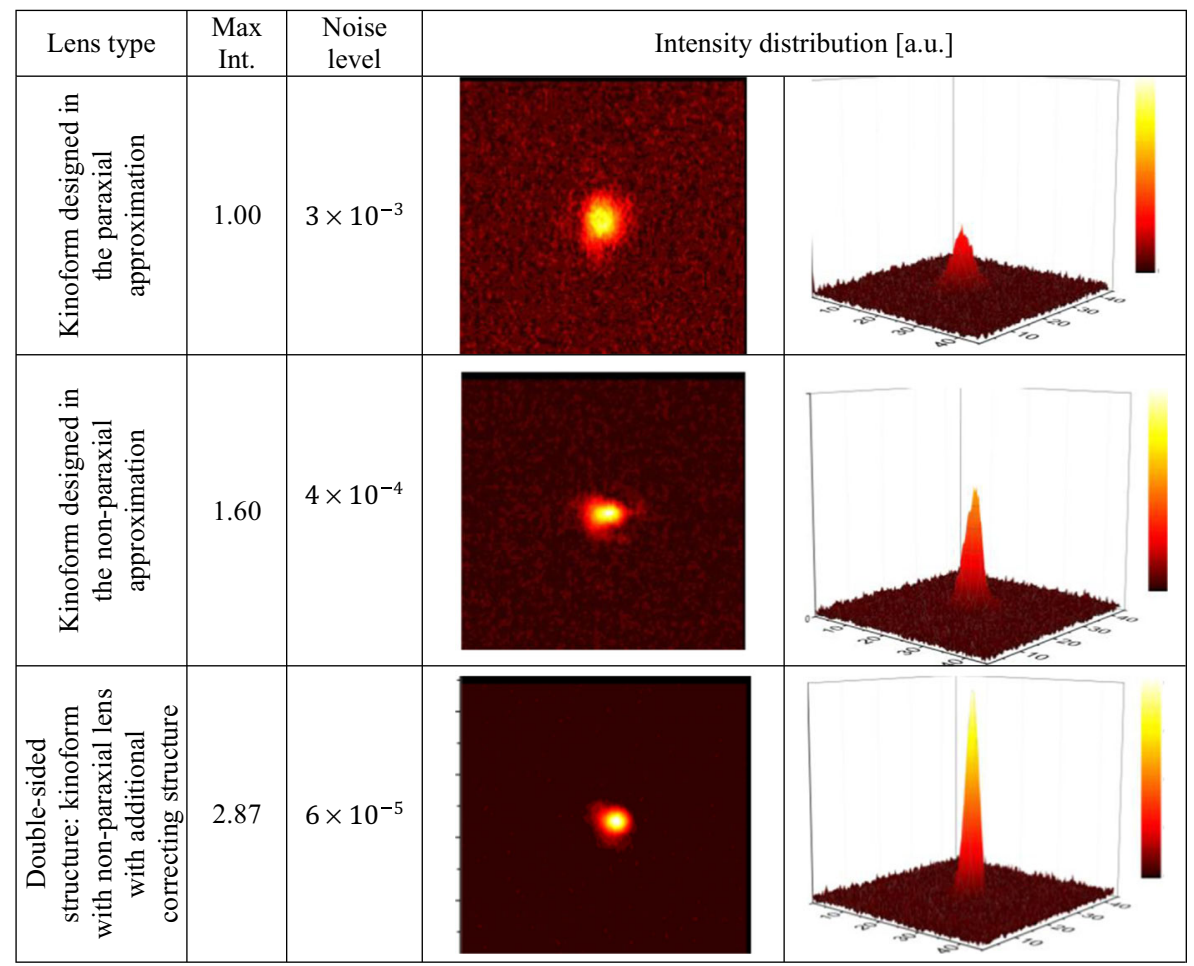

For the design wavelength $\mathrm{DWL}=1 \mathrm{~mm}$, the structure diameter $d=50 \mathrm{~mm}$ and the focal length $f=50 \mathrm{~mm}$ the diffraction limited focal spot has the diameter close to the $2.44 \mathrm{~mm}$. According to the theoretical simulations and experimental evaluation, the size of each focal spot was determined and presented in Table 2.

The maximal intensities shown in Table 1 were referred to the maximal intensity of the structure designed with the paraxial approximation that was normalised to 1 a.u. in order to facilitate the comparison of the achieved results. The non-paraxially designed structure has about $60 \%$ higher peak in the focal spot than the lens designed using the paraxial

Table 2 The comparison of focal spot sizes (diameters) for three types of lenses: the kinoform designed in the paraxial approximation, the kinoform designed in the non-paraxial approximation and the double-sided kinoform with non-paraxial lens with additional correcting structure

\begin{tabular}{lll}
\hline Type of lens & Theoretical spot size $[\mathrm{mm}] \begin{array}{c}\text { Experimental spot size } \\
{[\mathrm{mm}]}\end{array}$ \\
\hline $\begin{array}{l}\text { Kinoform designed in the paraxial approximation } \\
\text { Kinoform designed in the non-paraxial approxima- }\end{array}$ & 6.6 & 6.3 \\
$\quad$ tion & 3.6 & 5.4 \\
Double-sided structure: & 3.6 & 4.2
\end{tabular}


approximation. The kinoform created in the non-paraxial regime additionally equipped with a corrective phase structure enabled to increase the efficiency even of $187 \%$.

The intensity in the focal spot for lens designed in the paraxial approximation was measured at the limit of the sensitivity of the detector; hence, it is difficult to determine the real extent of the focal spot. However, both structures designed in the non-paraxial approximation (with and without correction) have similar size of the focal spot, which is close to the diffraction limit (which is also affected by illuminating the structure not with ideal planar wave). The power distribution of the focal spot formed by the lens with the correction is rounder and has sharper edges. In Table 2, the diameters of focal spots formed by three structures are compared (numerical simulation results with experimental data). According to the theory, the focal spot size of the lens limited with the aperture is defined as the diameter of Airy disc. Therefore, all values given in Table 2 are calculated between two points close to the noise level that lie on the straight line passing through the maximum intensity of the focal spot.

As we can see, the experimental spot sizes are a little bit bigger than data coming from theoretical simulations, but in the reality, the theoretical assumptions never can be fully fulfilled. Moreover, it is hard to unequivocally determine the place where the focal spot has its edge.

\section{Summary}

In this article, we proposed an innovative algorithm enabling to significantly suppress the geometrical aberration of the lens. To prove the rightness of the developed algorithm that corrects the diffractive structure, we have designed, manufactured and tested three types of DOEs: the kinoform designed in paraxial approximation, the kinoform designed in non-paraxial approach and double sided structure that consist of the kinoform designed without approximation and the additional correcting structure. Each of these structures was calculated using modified BPM and MCM techniques, they were manufactured by 3D printing and then experimentally evaluated in the optical setup using very sensitive and having small dimension Si MOSFET detectors.

In conclusion, the non-paraxial approach of designing DOEs having their apertures similar to their focal lengths gives better results than the paraxial approximation. Although both of these elements have good efficiency, it can be easily shown that adding the proposed correcting element significantly increases the efficiency of the lens-like element measured in its focal spot. Such compensating structures can be easily designed for more sophisticated optical elements than classical lens, which will allow to focus the radiation much more efficiently using lightweight, low cost and flat DOEs. Such correcting structures as proposed in this paper are attached on the other side of the already existing diffractive elements and have very small thickness in comparison to the whole structure; therefore, their absorption can be neglected due to the increase of efficiency they provide.

Open Access This article is distributed under the terms of the Creative Commons Attribution 4.0 International License (http://creativecommons.org/licenses/by/4.0/), which permits unrestricted use, distribution, and reproduction in any medium, provided you give appropriate credit to the original author(s) and the source, provide a link to the Creative Commons license, and indicate if changes were made. 


\section{References}

1. J. Richter, A. Hofmann, and L.-P. Schmidt, "Dielectric wide angle lenses for millimeter-wave focal plane imaging," Proceedings of the $31^{\text {st }}$ European Microwave Conference, London, UK, pp.347-350 (2001).

2. C. Bruckner, G. Notnia, and A. Tünnermann, "Optimal arrangement of 90 degrees off-axis parabolic mirrors in THz setups," Optik 121, 1, pp. 113-119, (2010).

3. E. D. Walsby, S. M. Durbin, D. R. S. Cumming, R. J. Blaikie, "Analysis of silicon terahertz diffractive optics," Curr. App. Phys. 4.2, pp.102-105, (2004).

4. O. Paul, B. Reinhard, B. Krolla, R. Beigang, M. Rahm, "Gradient index metamaterial based on slot elements," Appl. Phys. Lett. 96.24, 241110 (2010).

5. J. Suszek, A. Siemion, M. Bieda, N. Błocki; D. Coquillat; G. Cywiński; E. Czerwińska; M. Doch; A. Kowalczyk; N. Palka; A. Sobczyk; P. Zagrajek; M. Zaremba; A. Kolodziejczyk; W. Knap; M. Sypek "3-Dprinted flat optics for THz linear scanners," IEEE Thz Sci. T. 5, pp. 314-316 (2015).

6. M. Sypek, M. Makowski, E. Herault, Ag. Siemion, A. Siemion, J. Suszek, F. Garet, J-L Coutaz, "Highly efficient broadband double-sided Fresnel lens for THz range," Opt. Lett.,. 37.12, pp. 2214-2216 (2012)

7. M. Sypek, J.-L. Coutaz, A. Kolodziejczyk, M. Makowski, J. Suszek, "Aberrations of the large aperture attenuating THz lenses. Proceedings of Terahertz Technology and Applications V, (2012).

8. K. Wegrzynska, Ag. Siemion, M. Rachon, J. Suszek, M. Sypek, "Research on chromatic properties of high order kinoform," Proceedings of the Frontiers in Optics, San Jose, USA, (2015).

9. J. Suszek, A. M. Siemion, N. Błocki, M. Makowski, A. Czerwiński, J. Bomba, A. Kowalczyk, I. Ducin, K. Kakarenko, N. Pałka, P. Zagrajek, M. Kowalski, E. Czerwińska, C. Jastrzebski, K. Świtkowski, J.-L. Coutaz, A. Kolodziejczyk, M. Sypek "High order kinoforms as a broadband achromatic diffractive optics for terahertz beams,” Opt. Express 22.3, pp. 3137-3144 (2014).

10. P. Wang, N. Mohammad, R. Menon, "Chromatic-aberration-corrected diffractive lenses for ultra-broadband focusing,"Sci. Report. 6, (2016).

11. J.W. Goodman, Introduction to Fourier Optics, chapter 3-6, 10 (pp. 31-173, 399-427), W. H. Freeman; 3 edition (December 10, 2004)

12. J. A. Jr. Fleck, J. R. Morris, M. D. Feit, "Time-Dependent Propagation of High Energy Laser Beams through the Atmosphere," App. Phys., 10.2, pp. 129-160 (1976).

13. M. Sypek, "Light propagation in the Fresnel Region. New numerical approach," Opt. Commun., 116.1, pp. 43-48 (1995).

14. Z. Jaroszewicz, A. Kołodziejczyk, M. Sypek, C. Gomez-Reino, "Non-paraxial analytical solution for the generation of focal curves," J. Mod. Opt., 43.3, pp.617-637 (1996).

15. F. Schuster, D. Coquillat, H. Videlier, M. Sakowicz, F. Teppe, L. Dussopt, B. Giffard, T. Skotnicki, and W. Knap, "Broadband terahertz imaging with highly sensitive silicon CMOS detectors," Opt. Express 19.8, pp. 7827-7832 (2011). 